\title{
Nanoscale
}

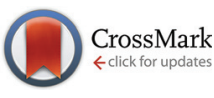

Cite this: Nanoscale, 2015, 7, 9963

\section{Processes for non-destructive transfer of graphene: widening the bottleneck for industrial scale production}

\begin{abstract}
Aliaksandr V. Zaretski and Darren J. Lipomi*
The exceptional charge-transport, mechanical, and barrier properties of graphene are well known. Highquality films of single-layer graphene produced over large areas, however, are extremely expensive. The high cost of graphene precludes its use in industries-such as transparent electrodes and flexible packaging-that might take full advantage of its properties. This minireview presents several strategies for the transfer of graphene from the substrates used for growth to substrates used for the final application. Each strategy shares the characteristic of being non-destructive: that is, the growth substrate remains reusable for further synthesis of new graphene. These processes have the potential to lower significantly the costs of manufacturing graphene, to increase production yields, and to minimize environmental impact. This article is divided into sections on (i) the synthesis of high-quality single-layer graphene and (ii) its nondestructive transfer to a host substrate. Section (ii) is further divided according to the substrate from which graphene is transferred: single-crystalline wafers or flexible copper foils. We also comment, wherever possible, on defects produced as a result of the transfer, and potential strategies to mitigate these defects. We conclude that several methods for the green synthesis and transfer of graphene have several of the right characteristics to be useful in industrial scale production.
\end{abstract}

Received 19th March 2015

Accepted 15th April 2015

DOI: $10.1039 / c 5 n r 01777 g$

www.rsc.org/nanoscale production of graphene, that is, inexpensive, non-destructive transfer from the substrate used for synthesis to the final substrate used for the application. The current state-of-the-art techniques for transferring high-quality single layers are slow (i.e., they cost a minimum of $30 \mathrm{~min}$ per batch because of etching the growth substrate) and deleterious to the environment. ${ }^{8}$ To enable proliferation of large-area graphene to every industry for which the material has potential-and potentially great-value, the ability to transfer graphene rapidly and in a non-destructive manner is required. While there exist methodologies of inexpensive large-volume production of suspensions of exfoliated graphene that can be spray-coated onto a substrate to generate conducting surfaces ${ }^{9}$ or mixed in with polymers to make conductive composites ${ }^{10}$ and barrier films, ${ }^{11}$ this minireview focuses only on processes for transfer that preserve the desirable characteristics of high-quality single-layer graphene, including high transparency, high electron mobility, and low sheet resistance. ${ }^{12}$

\section{Synthesis of large-area graphene}

There are two principal methodologies for the synthesis of large-area single-layer graphene: silicon-carbide (SiC) epitaxy ${ }^{13}$ and chemical vapor deposition (CVD) ${ }^{14}$ While neither of the techniques guarantees the formation of a single-layer of gra-
Department of NanoEngineering, University of California, San Diego, 9500 Gilman Drive Mail Code 0448, La Jolla, CA 92093-0448, USA. E-mail: dlipomi@ucsd.edu 
a
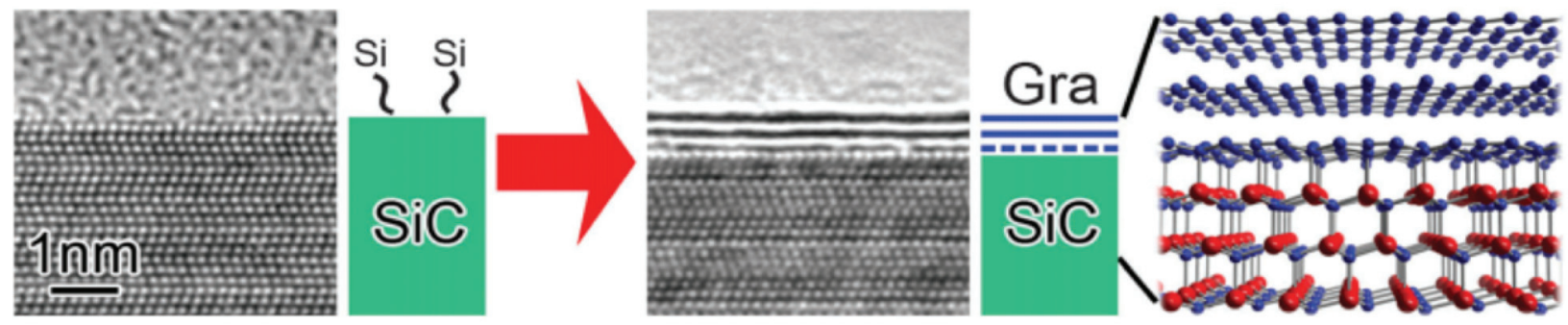

b
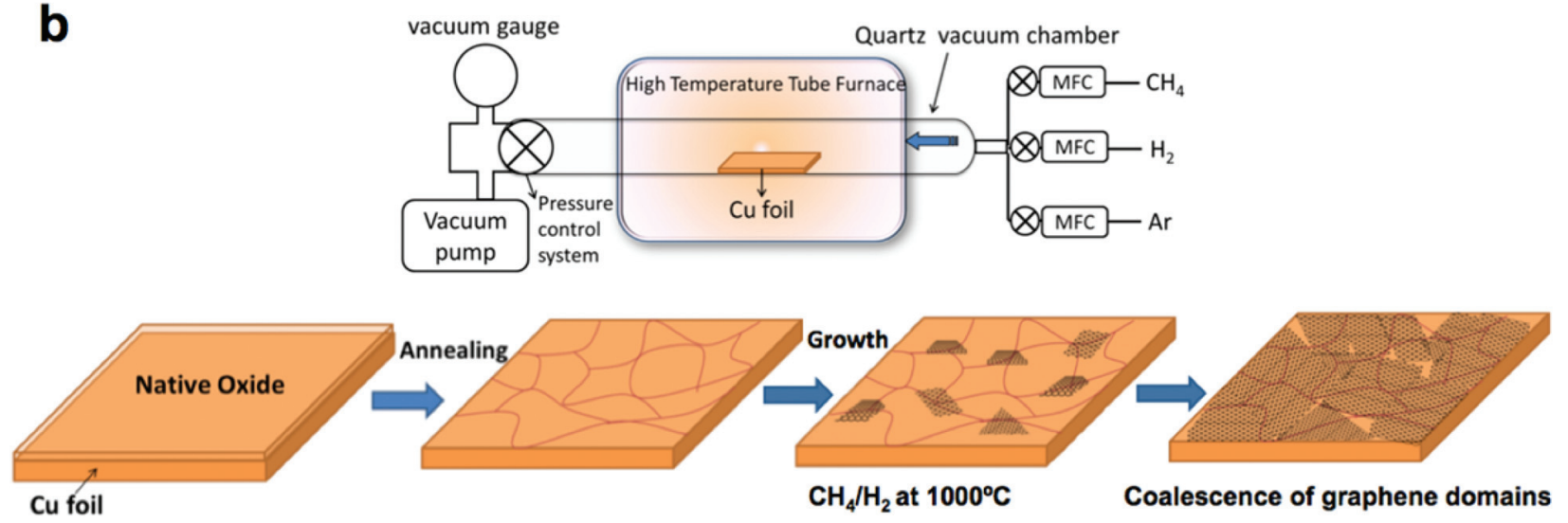

Fig. 1 Methods for synthesizing high-quality large-area graphene. (a) Epitaxial synthesis of graphene on a SiC wafer. Reproduced with permission from ref. 13. Copyright 2014, Royal Society of Chemistry. (b) Chemical vapor deposition (CVD) of graphene on copper foil. Reproduced with permission from ref. 16, Copyright 2011, American Chemical Society, and from ref. 20, Copyright 2013, InTech.

phene, both can be modified to do so, and both have their advantages and limitations.

\subsection{SiC epitaxy}

In $\mathrm{SiC}$ epitaxy, a single crystalline $\mathrm{SiC}$ wafer is heated in a vacuum or argon atmosphere to the temperatures above $2000{ }^{\circ} \mathrm{C}$. At this temperature, silicon atoms sublimate from the (0001) face of the crystal. The remaining carbon (which has a lower vapor pressure than silicon) rearranges to form graphene (Fig. 1a). ${ }^{13}$ By tight control of the parameters of this process, synthesis of single-, few-, and multilayer graphene has been demonstrated. ${ }^{15}$ One particular advantage of this method is that it produces graphene covering the entire surface of an insulating wafer, and thus allows the fabrication of circuitry directly on the growth substrate. Such graphene possesses exceptionally high electron mobilities, which is desirable for high-performance components of integrated circuits. We return to this process in section 3.1.1 when we discuss the process of transferring single layer graphene from the $\mathrm{SiC}$ wafer onto flexible host substrates.

\subsection{CVD of graphene}

The process of chemical vapor deposition (CVD) of graphene offers great versatility as it allows synthesis of single- or multilayer graphene on a large number of substrates (usually refractory metals, ${ }^{16}$ Fig. $1 \mathrm{~b}$ ). The use of copper foil is by far the best studied, because copper is relatively inexpensive. Moreover, the low solubility of carbon in copper permits synthesis of predominantly single layer graphene of high quality. ${ }^{16} \mathrm{CVD}$ of graphene can be performed in vacuum ${ }^{17}$ or atmospheric pressure ${ }^{18}$ and at temperatures as low as $300{ }^{\circ} \mathrm{C}^{19}$ Additionally, as compared to SiC epitaxy, CVD of graphene is not limited to the small dimensions of a wafer.

\section{Graphene transfer methods}

In order to be usable, the graphene synthesized on copper foil by CVD has to be transferred to the substrate of interest, which usually requires supporting of graphene by a polymeric film and etching the copper foil in a corrosive medium ${ }^{8,20}$ (Fig. 2). Such transfer processes are time consuming: it takes at least $30 \mathrm{~min}$ to dissolve $25 \mu \mathrm{m}$-thick copper foil. This process is also wasteful: it requires $300 \mathrm{~kg}$ of copper to produce $1 \mathrm{~g}$ of graphene, ${ }^{21}$ and thus produces an outsized amount of toxic waste. These disadvantages currently limit the fabrication of graphene to small amounts mostly for research and development purposes and need to be overcome in order to make graphene over large areas at low costs.

\subsection{Non-destructive graphene transfer}

In order to remove graphene from the synthesis substrate non-destructively so that the substrate can be recycled, the 

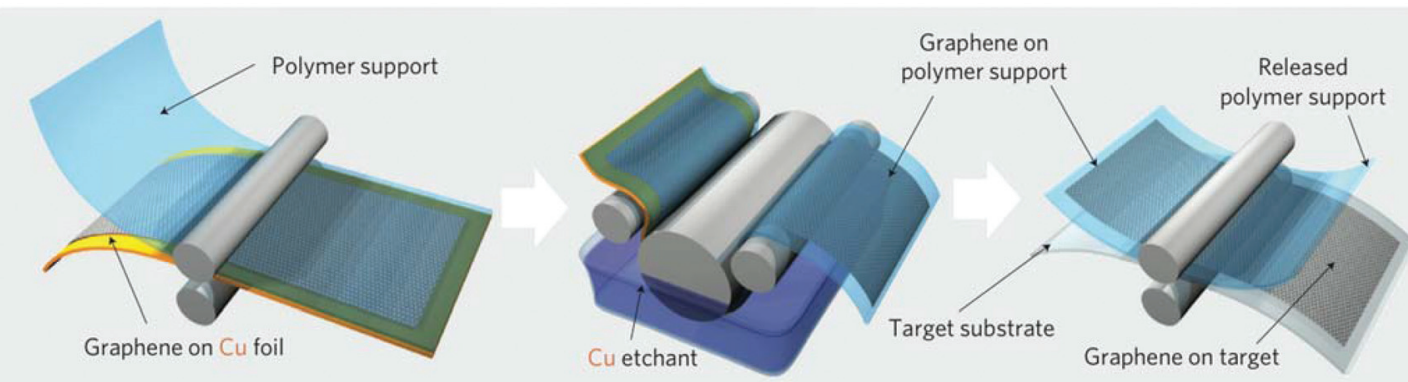

Fig. 2 Schematic of the wet roll-to-roll graphene transfer from copper foils to polymeric substrates. Reproduced with permission from ref. 8. Copyright 2010, Nature Publishing Group.

adhesion between graphene and the growth substrate needs to be overcome. Despite its mechanical strength, a single-layer of graphene is too delicate to be exfoliated unsupported, or manipulated as a free-standing film, and thus requires a relatively rigid backing. Typically, the rigid support is a thicker polymeric sheet or foil that, in some cases, is the final receiving substrate, or is an intermediate substrate used only for mechanical support during transfer. In any case, the strength of adhesion of graphene to the supporting substrate has to be greater than to the substrate used for growth. The supporting substrate can provide strong adhesion in either its native state or it can be modified with adhesion layers whose effect is produced by covalent or van der Waals bonding. Knowing both values of adhesion energy, or at least their relative magnitudes, allows the design of the graphene exfoliation process from a given growth substrate to the final receiving substrate either directly or using a multi-step manipulation. The strengths of adhesion of graphene to various media are listed in Table 1.

The design of a process to exfoliate graphene is not limited to the relative strengths of adhesion between graphene and the substrate used for synthesis and the substrate used for exfoliation. For example, the roughness of the substrate used for growth and the parameters of processing-e.g., the temperature and pressure at which the supporting substrate is deposited, which affects the interfacial area between the graphene and the supporting substrate-also plays a key role. A hot press is often used to apply the supporting substrate to increase its adhesion to the graphene. Another consideration

Table 1 Strength of adhesion of graphene to growth/support substrates

\begin{tabular}{lll}
\hline Interface & $\begin{array}{l}\text { Strength of } \\
\text { adhesion }\left(\mathrm{J} \mathrm{m}^{-2}\right)\end{array}$ & Ref. \\
\hline Graphene/graphene & 0.3 & 22 \\
Graphene/copper & $0.4-0.7$ & $23-25$ \\
Graphene/gold & 0.7 & 26 \\
Graphene/SiC & $2.3-3.0$ & 27 and 28 \\
Graphene/nickel & 3.5 & 23
\end{tabular}

is the chemical modification of graphene by the supporting/ adhesion layer (covalent bonding, doping), which can potentially change the electronic properties of graphene. ${ }^{29}$ Not least important are the mechanical aspects of the transfer process such as the distribution of stress during the exfoliation, which may be especially problematic with roll-to-roll transfer. For example, a film that is 200 microns thick when bent to the radius of 5 millimeters experiences a tensile strain on the top surface of $2 \%$ and an equal in magnitude compressive strain on the bottom surface. ${ }^{30}$ Such strains can cause catastrophic failures in a number of thin-film materials. ${ }^{31}$

3.1.1 Graphene transfer from single-crystalline wafers. As discussed in section 2.1, SiC epitaxy is a useful technique for generating high-quality graphene with a high degree of control over the number of graphene layers and their crystallographic orientations. ${ }^{15}$ Additionally, graphene synthesized in this fashion exhibits minimal surface roughness. Considering the weak graphene/graphene interlayer interaction (Table 1), it is easily exfoliated from a multilayer stack. ${ }^{32}$ As demonstrated by Kim et al., ${ }^{33}$ however, even single-layer graphene, which is relatively strongly bonded to SiC, can be exfoliated with the right adhesion layer. Using an evaporated thin nickel film as an adhesion layer and thermal-release tape as the supporting substrate, the authors were able to exfoliate single-layer graphene (with some graphene add-layers) from the $\mathrm{SiC}$ wafer by direct fracture of the SiC/graphene interface. The authors "cleaned" the transferred graphene-i.e., removed the add-layers-by evaporating and subsequently exfoliating a thin film of gold, along with the noncontiguous sheets of the double layers. The choice of gold was justified because its adhesion to graphene is stronger than graphene to graphene, but weaker than nickel to graphene. The resulting graphene appeared contiguous and exhibited a minimal D-peak in the Raman spectrum. The low intensity of this signal was consistent with a low density of defects (Fig. 3a). This nickel-supported graphene could also be transferred onto the final receiving substrate $\left(\mathrm{Si} / \mathrm{SiO}_{2}\right.$ wafer $)$ after which the metal could easily be etched away. After exfoliation of graphene, the $\mathrm{SiC}$ wafer was immediately available for an additional cycle of graphene synthesis. Besides providing the necessary adhesion strength, the evaporated nickel film 

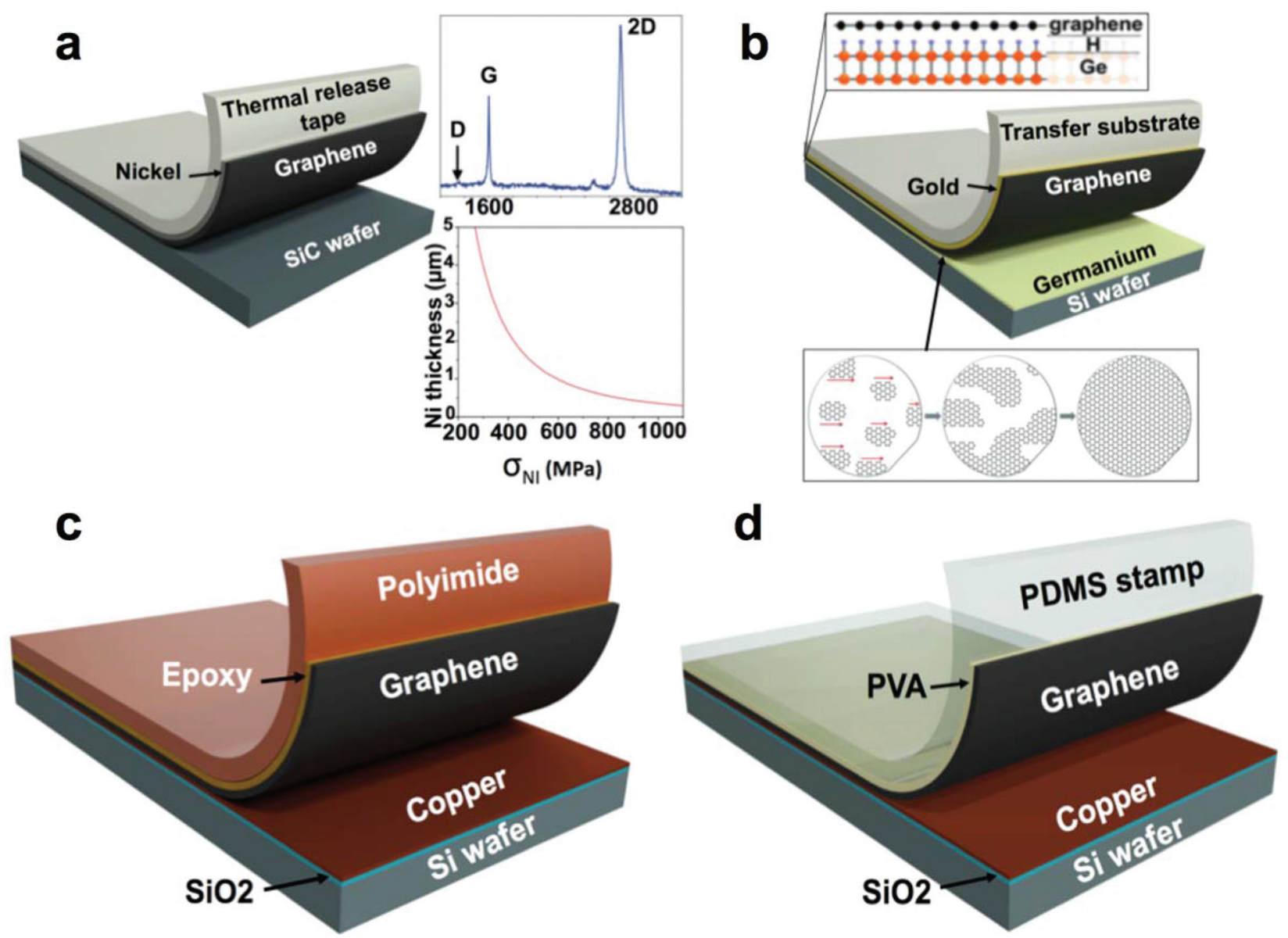

Fig. 3 Summary of methods used to transfer graphene from single-crystalline wafers. (a) Schematic diagram of graphene transfer from SiC wafer after SiC epitaxy. Reproduced with permission from ref. 33. Copyright 2013, American Association for the Advancement of Science. (b) Graphene transfer from a silicon wafer bearing a film of germanium. Reproduced with permission from ref. 35. Copyright 2014, American Association for the Advancement of Science. (c) Graphene transfer from evaporated copper substrate using epoxy. Adapted from ref. 25. (d) Graphene transfer from evaporated copper substrate using a PDMS/PVA stamp. Adapted from ref. 36.

imposes epitaxial stress onto graphene. The additional stress facilitated adhesive fracture of the copper/graphene interface during the exfoliation. ${ }^{34}$

Repetitive synthesis by CVD and subsequent exfoliation of a wafer-scale single-crystalline single-layer graphene from a hydrogen-terminated germanium-coated silicon wafer has been demonstrated by Lee et $a l^{35}$ This process was facilitated by three characteristics (1) the low solubility of carbon in germanium, (2) its catalytic activity toward methane cracking, and (3) its perfect crystalline order as deposited on the surface of the silicon wafer. The hydrogen-terminated germanium, which exhibited a weak adhesion to graphene, allowed the authors to exfoliate the graphene from the wafer using a thin film of evaporated gold (Fig. 3b). This group also demonstrated a repetitive sequential graphene synthesis and exfoliation from the same wafer without any apparent deterioration of the graphene. ${ }^{35}$

Copper evaporated onto a silicon wafer bearing a layer of thermally grown oxide can also support CVD of graphene. Yoon et $a .^{25}$ demonstrated the effective direct exfoliation of graphene from the copper-coated wafer to a flexible polyimide film (PI) by binding them with epoxy as the permanent adhesion layer (Fig. 3c). The authors stated that this approach helped to avoid doping of graphene with metals and the detrimental acidic treatments. They demonstrated the utility of the graphene prepared in this manner by fabricating a flexible field-effect transistor (FET) with good gate modulation of the graphene channel conductivity on the PI film. Regrowth of graphene on the same substrate was performed and generated graphene with a somewhat increased defect density as judged by the Raman D-peak of graphene on copper. ${ }^{25}$ This defectiveness could be explained in principle by the evaporative deterioration of the thin copper layer during graphene synthesis.

Similarly, Yang et al. $^{36}$ employed an adhesion layer, albeit water soluble-polyvinyl alcohol-to exfoliate graphene from copper-coated $\mathrm{Si} / \mathrm{SiO}_{2}$ wafer and to transfer it to arbitrary substrates (Fig. 3d). During the transfer, graphene sustained minimal physical damage, as indicated by the $\mathrm{D} / \mathrm{G}$ peak ratio of 0.06. A polydimethylsiloxane (PDMS) stamp served as a 
support substrate for the transfer and provided a kinetically controlled adhesion necessary for the graphene transfer: rapidly peeling the stamp away from the copper-coated wafer promoted exfoliation of the PVA-coated graphene. In the next step, the PVA-coated graphene was pressed against the final receiving substrate and by peeling the stamp away slowly was released. After the transfer, the PVA adhesion layer could be dissolved in deionized water. As with the exfoliation process that did not involve metal etching, developed by Yoon et al. ${ }^{25}$ above, the graphene produced by this method was free of doping and preserves its charge neutrality with the value of $V_{\text {dirac }}$ close to $0 \mathrm{~V}$ in the FETs fabricated with the exfoliated graphene.

3.1.2 Graphene transfer from copper foil. Certain applications require the synthesis of graphene over areas greater than are possible using the largest available single crystalline wafers ( $\leq 12$ inches in diameter). These applications include transparent conducting electrodes for liquid crystal displays, photovoltaic panels, and barrier films for electronics and flexible packaging. So far, the only method that can generate equal or greater areas of single-layer and high-quality material is CVD on copper foil. Exfoliation of graphene is especially challenging due to the intrinsic surface roughness and highly pliable nature of the copper foil after annealing. Due to the manufacturing process (cold-rolling), commercially available copper foils have grooves that, in turn, produce highly anisotropic rms roughness of $615 \mathrm{~nm} .{ }^{18}$ Even when the copper foils are electropolished and the roughness is reduced to $148 \mathrm{~nm},{ }^{18}$ after graphene synthesis, >150 nm-deep fissures form at the copper grain boundaries. ${ }^{37}$ In case of atomically smooth copper films produced by deposition and stripping from a single-crystalline wafer, heating to $1000{ }^{\circ} \mathrm{C}$ increases the roughness from $0.5 \mathrm{~nm}$ to above $10 \mathrm{~nm} .{ }^{19}$ Hence the exfoliation process designed for removing graphene from copper foils needs to accommodate the roughness. Several groups have designed such processes that employ a polymeric receiving substrate and a hot press that heats the polymer above its glass transition temperature, while the high pressure molds the polymer into the relief structures in the copper foil. In this way, Fechine et $a l .{ }^{38}$ demonstrated a complete graphene trans-
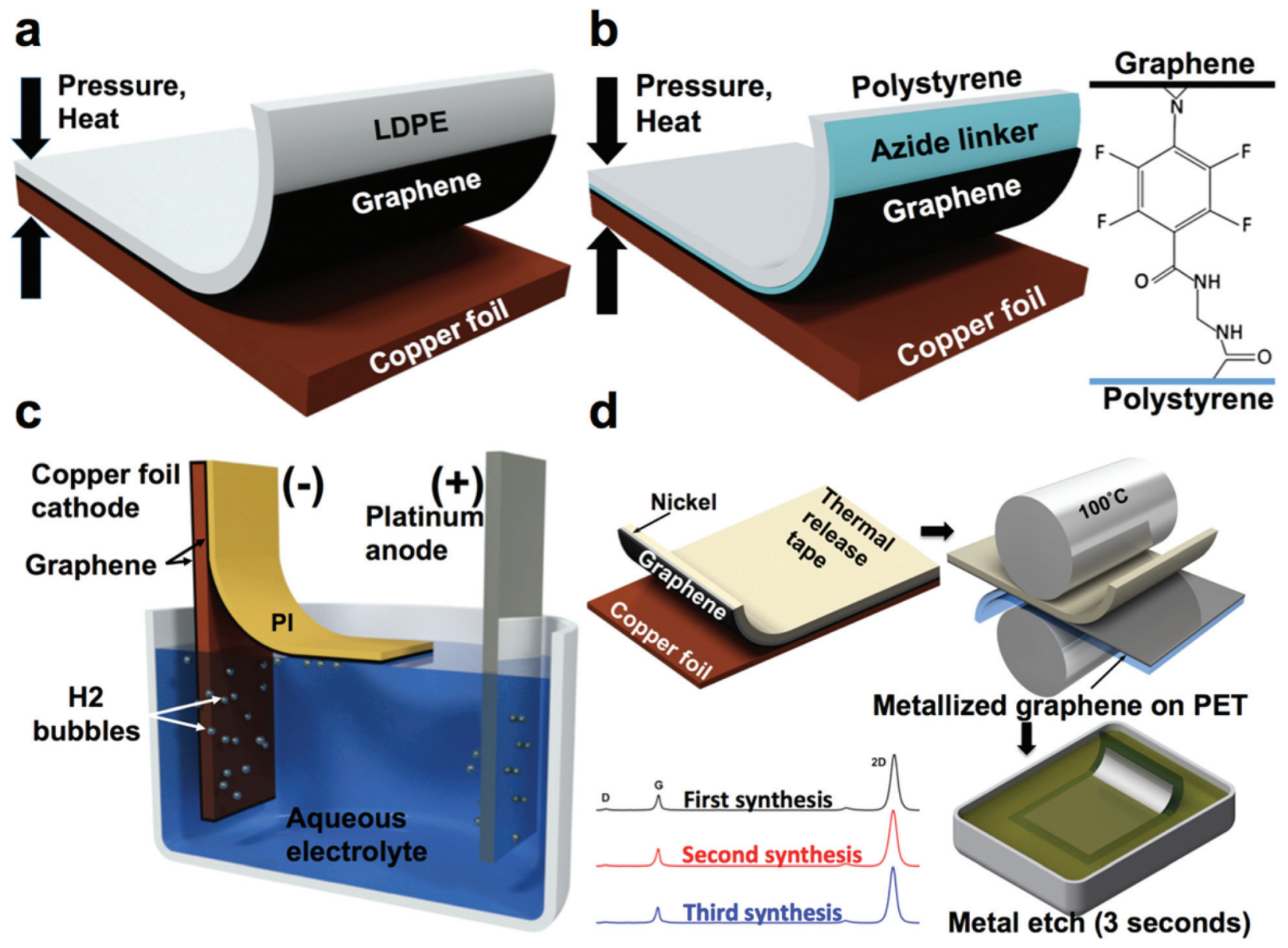

Fig. 4 Summary of methods used to transfer graphene from flexible copper foils. (a) Direct dry transfer of graphene from copper foil to polymeric substrates using a hot press. Adapted from ref. 38. (b) Adhesion molecule assisted dry transfer of graphene from copper foil to polymeric substrates using a hot press. Adapted from ref. 39. (c) Electrochemical delamination of PI-supported graphene from copper foil. Adapted from ref. 43. (d) Metal-assisted exfoliation of graphene from copper foil. Adapted from ref. 21. 
fer (albeit of an unknown graphene intactness) from a copper foil to low-density polyethylene (LDPE) and to other polymers (Fig. 4a).

A similar approach enhanced with a molecule that covalently bonds graphene to polystyrene- $\mathrm{N}$-ethylamino-4azidotetrafluorobenzoate (TFPA- $\mathrm{NH}_{2}$ ) -was employed by Lock et $a .^{39-41}$ and resulted in graphene with a sheet resistance of about $1 \mathrm{k} \Omega \mathrm{sq}^{-1}$ (Fig. 4b). TFPA is known to form strong covalent bonds with graphene ${ }^{42}$ and thus the polystyrene substrate was dip-coated in TFPA- $\mathrm{NH}_{2}$ solution after plasma treatment to promote the adhesion to graphene. It is noteworthy that without the TFPA treatment, polystyrene demonstrated poor adhesion, which resulted in only partial (19\%) transfer. ${ }^{38}$

A noteworthy process to transfer graphene non-destructively by "electrochemical delamination" has been demonstrated by Wang et al. ${ }^{43}$ In this process, the final receiving substrate (PI) is spin-coated on the CVD graphene on copper foil, which serves as a cathode in an electrochemical cell with $0.5 \mathrm{M}$ sodium hydroxide as an electrolyte and platinum wire mesh as an anode. By applying a $15 \mathrm{~V}$ bias between the electrodes, hydrolysis of water produced hydrogen bubbles between the copper foil and graphene and promoted separation of this interface (Fig. 4c). Graphene supported by $9 \mu \mathrm{m}$-thick PI was observed to float away from the copper foil. It was then collected, and its conductivity was measured under various bending radii. The process reportedly took $5 \mathrm{~min}$ for a $50 \times$ $15 \mathrm{~mm}$ size sheet and generated graphene with a sheet resistance of $459 \Omega \mathrm{sq}^{-1}$.

Our laboratory reported a process for exfoliating single-layer graphene from copper foils without the use of a hot press, nicknamed metal-assisted exfoliation (MAE, Fig. 4d). ${ }^{21}$ This method employed the evaporation of a thin film $(>20 \mathrm{~nm})$ of nickel or cobalt onto the CVD-grown graphene on copper foil. The preferential adhesion of graphene to nickel and cobalt ensured delamination of graphene from the copper during exfoliation, while the use of a thermal-release tape as the supporting substrate enabled the transfer of the metalized exfoliated graphene onto flexible transparent polymeric films bearing a thermoplastic adhesive by quick processing through a commercial document laminator. Further, the nanoscopic metal film (nickel or cobalt) could be etched rapidly in an acidic solution to yield single-layer graphene on a flexible polymeric film.

The mechanical stresses induced in thin films during exfoliation process need to be considered as even small forces may cause large deformations. Evidence of the effects of anisotropic stresses induced in roll-to-roll processing is the anisotropic sheet resistances of the graphene resulting from MAE when excessively large tensile strains are imposed onto the metallized graphene during the process of exfoliation. The bending radii of the metallized graphene supported by thermal release tape during the manual exfoliation were small enough to cause metal film cracking that propagates through graphene and produced sheet resistances on the order of $0.8 \mathrm{k} \Omega \mathrm{sq}^{-1}$ parallel to the cracks and $8 \mathrm{k} \Omega \mathrm{sq}^{-1}$ in the perpendicular orientation. This problem can in principle be solved by avoiding excessively small bending radii during the exfoliation process or by using very thin supporting substrates.

\section{Conclusion}

With more than 8000 patents filed to date, graphene has been proposed for a multitude of possible applications in a number of various industries. It is likely that different approaches of graphene synthesis and transfer will be used for different applications, considering the strong dependence of the quality, yield, and cost of graphene based on the process selected. Some of the processes discussed in this minireview may be better suited for research and development and small chip-scale production, while others are more amenable to industrial-scale roll-to-roll fabrication schemes. Further developments are needed in order to overcome the still remaining shortcomings: mechanical damage during exfoliation, the use of expensive sacrificial layers (evaporated metal films) and transfer substrates (thermal-release tapes and thermoplastic adhesives). In particular, routes of mechanical degradation of the graphene should be characterized more fully than they currently are, so that these pathways can be mitigated. We conclude that non-destructive, economically feasible, and environmentally friendly and sustainable processes for manufacturing graphene at scales demanded by industry will need to be further developed, though several potentially significant strategies have been proposed. Progress in these areas is required if this material is to make a truly significant impact on society.

\section{Acknowledgements}

This work was supported by the laboratory startup funds from the University of California, San Diego. A. Z. acknowledges a fellowship from SoCal Clean Energy Technology Acceleration Program from the von Liebig Center at UCSD sponsored by the US Department of Energy, and a fellowship from the National Science Foundation Graduate Research Fellowship Program, DGE-1144086.

\section{References}

1 I. Khrapach, F. Withers, T. H. Bointon, D. K. Polyushkin, W. L. Barnes, S. Russo and M. F. Craciun, Adv. Mater., 2012, 24, 2844-2849.

2 J. Ryu, Y. Kim, D. Won, N. Kim, J. S. Park, E.-K. Lee, D. Cho, S.-P. Cho, S. J. Kim, G. H. Ryu, H.-A.-S. Shin, Z. Lee,

B. H. Hong and S. Cho, ACS Nano, 2014, 8, 950-956.

3 D. E. Jiang, V. R. Cooper and S. Dai, Nano Lett., 2009, 9, 4019-4024.

4 Z. Liu, J. Li and F. Yan, Adv. Mater., 2013, 25, 4296-4301.

5 K. S. Novoselov, A. K. Geim, S. V. Morozov, D. Jiang, Y. Zhang, S. V. Dubonos, I. V. Grigorieva and A. A. Firsov, Science, 2004, 306, 666-669. 
6 T. Kobayashi, M. Bando, N. Kimura, K. Shimizu, K. Kadono, N. Umezu, K. Miyahara, S. Hayazaki, S. Nagai, Y. Mizuguchi, Y. Murakami and D. Hobara, Appl. Phys. Lett., 2013, 102, 023112.

7 T. Yamada, J. Kim, M. Ishihara and M. Hasegawa, J. Phys. D: Appl. Phys., 2013, 46, 063001.

8 S. Bae, H. Kim, Y. Lee, X. Xu, J.-S. Park, Y. Zheng, J. Balakrishnan, T. Lei, H. R. Kim, Y. Il Song, Y.-J. Kim, K. S. Kim, B. Ozyilmaz, J.-H. Ahn, B. H. Hong and S. Iijima, Nat. Nanotechnol., 2010, 5, 574-578.

9 D.-Y. Kim, S. Sinha-Ray, J.-J. Park, J.-G. Lee, Y.-H. Cha, S.-H. Bae, J.-H. Ahn, Y. C. Jung, S. M. Kim, A. L. Yarin and S. S. Yoon, Adv. Funct. Mater., 2014, 24, 4986-4995.

10 Y. Li, Y. A. Samad, K. Polychronopoulou, S. M. Alhassan and K. Liao, Sci. Rep., 2014, 4, 4652.

11 Y. Su, V. G. Kravets, S. L. Wong, J. Waters, A. K. Geim and R. R. Nair, Nat. Commun., 2014, 5, 4843.

12 J. S. Bunch, S. S. Verbridge, J. S. Alden, A. M. Van Der Zande, J. M. Parpia, H. G. Craighead and P. L. McEuen, Nano Lett., 2008, 8, 2458-2462.

13 W. Norimatsu and M. Kusunoki, Phys. Chem. Chem. Phys., 2014, 16, 3501-3511.

14 A. Reina, X. Jia, J. Ho, D. Nezich, H. Son, V. Bulovic, M. S. Dresselhaus and J. Kong, Nano Lett., 2008, 9, 30-35.

15 W. Norimatsu and M. Kusunoki, Chem. Phys. Lett., 2009, 468, 52-56.

16 C. Mattevi, H. Kim and M. Chhowalla, J. Mater. Chem., 2011, 21, 3324.

17 N. S. Mueller, A. J. Morfa, D. Abou-Ras, V. Oddone, T. Ciuk and M. Giersig, Carbon N. Y., 2014, 78, 347-355.

18 I. Vlassiouk, P. Fulvio, H. Meyer, N. Lavrik, S. Dai, P. Datskos and S. Smirnov, Carbon N. Y., 2013, 54, 58-67.

19 P. Procházka, J. Mach, D. Bischoff, Z. Lišková, P. Dvořák, M. Vaňatka, P. Simonet, A. Varlet, D. Hemzal, M. Petrenec, L. Kalina, M. Bartošík, K. Ensslin, P. Varga, J. Čechal and T. Šikola, Nanotechnology, 2014, 25, 185601.

20 A. Kumar and C. H. Lee, in Advances in Graphene Science, ed. M. Aliofkhazraei, 2013, pp. 55-75. DOI: 10.5772/55728.

21 A. V. Zaretski, H. Moetazedi, C. Kong, E. J. Sawyer, S. Savagatrup, E. Valle, T. F. O'Connor, A. D. Printz and D. J. Lipomi, Nanotechnology, 2015, 26, 45301.

22 D. J. Henry, G. Yiapanis, E. Evans and I. Yarovsky, J. Phys. Chem. B, 2005, 109, 17224-17231.

23 J. Lahiri, T. S. Miller, A. J. Ross, L. Adamska, I. I. Oleynik and M. Batzill, New J. Phys., 2011, 13, 025001.

24 Z. Xu and M. J. Buehler, J. Phys.: Condens. Matter, 2010, 22, 485301.

25 T. Yoon, W. C. Shin, T. Y. Kim, J. H. Mun, T.-S. Kim and B. J. Cho, Nano Lett., 2012, 12, 1448-1452.

26 I. Hamada and M. Otani, Phys. Rev. B: Condens. Matter Mater. Phys., 2010, 82, 153412.
27 A. Mattausch and O. Pankratov, Phys. Rev. Lett., 2007, 99, 086802.

28 G. H. Wells, T. Hopf, K. V. Vassilevski, E. Escobedo-Cousin, N. G. Wright, A. B. Horsfall, J. P. Goss, A. G. O'Neill and M. R. C. Hunt, Appl. Phys. Lett., 2014, 105, 193109.

29 H. Liu, Y. Liu and D. Zhu, J. Mater. Chem., 2011, 21, 3335.

30 N. Lu, Z. Suo and J. J. Vlassak, Acta Mater., 2010, 58, 16791687.

31 S. Savagatrup, A. D. Printz, T. F. O'Connor, A. V. Zaretski, D. Rodriquez, E. J. Sawyer, K. M. Rajan, R. I. Acosta, S. E. Root and D. J. Lipomi, Energy Environ. Sci., 2015, 8, 55-80.

32 T. Moldt, A. Eckmann, P. Klar, S. V. Morozov, A. A. Zhukov, K. S. Novoselov and C. Casiraghi, ACS Nano, 2011, 5, 77007706.

33 J. Kim, H. Park, J. B. Hannon, S. W. Bedell, K. Fogel, D. K. Sadana and C. Dimitrakopoulos, Science, 2013, 342, 833-836.

34 Y. He, W. F. Chen, W. B. Yu, G. Ouyang and G. W. Yang, Sci. Rep., 2013, 3, 2660.

35 J.-H. Lee, E. K. Lee, W.-J. Joo, Y. Jang, B.-S. Kim, J. Y. Lim, S.-H. Choi, S. J. Ahn, J. R. Ahn, M.-H. Park, C.-W. Yang, B. L. Choi, S.-W. Hwang and D. Whang, Science, 2014, 344, 286-289.

36 S. Y. Yang, J. G. Oh, D. Y. Jung, H. Choi, C. H. Yu, J. Shin, C.-G. Choi, B. J. Cho and S.-Y. Choi, Small, 2015, 11, 175181.

37 D. Lee, G. D. Kwon, J. H. Kim, E. Moyen, Y. H. Lee, S. Baik and D. Pribat, Nanoscale, 2014, 6, 12943-12951.

38 G. J. M. Fechine, I. Martin-Fernandez, G. Yiapanis, R. Bentini, E. S. Kulkarni, R. V. Bof de Oliveira, X. Hu, I. Yarovsky, A. H. Castro Neto and B. Özyilmaz, Carbon N. Y., 2015, 83, 224-231.

39 E. H. Lock, M. Baraket, M. Laskoski, S. P. Mulvaney, W. K. Lee, P. E. Sheehan, D. R. Hines, J. T. Robinson, J. Tosado, M. S. Fuhrer, S. C. Hernández and S. G. Walton, Nano Lett., 2012, 12, 102-107.

40 E. H. Lock, D. M. Delongchamp, S. W. Schmucker, B. Simpkins, M. Laskoski, S. P. Mulvaney, D. R. Hines, M. Baraket, S. C. Hernandez, J. T. Robinson, P. E. Sheehan, C. Jaye, D. A. Fisher and S. G. Walton, Carbon N. Y., 2015, 86, 288-300.

41 E. H. Lock, S. C. Hernández, T. J. Anderson, S. W. Schmucker, M. Laskoski, S. P. Mulvaney, F. J. Bezares, J. D. Caldwell, P. E. Sheehan, J. T. Robinson, B. N. Feygelson and S. G. Walton, Surf. Coat. Technol., 2014, 241, 118-122.

42 L. H. Liu and M. Yan, Nano Lett., 2009, 9, 3375-3378.

43 X. Wang, L. Tao, Y. Hao, Z. Liu, H. Chou, I. Kholmanov, S. Chen, C. Tan, N. Jayant, Q. Yu, D. Akinwande and R. S. Ruoff, Small, 2014, 10, 694-698. 